\title{
Trichinose expérimentale
}

\section{$\left(3^{\mathrm{e}}\right.$ note $)$}

\section{Transplantations intestinales de Tricbinella spiralis adultes}

\author{
par Geneviève MOUGEOT et Félix LANCASTRE
}

Laboratoire de Parasitologie, «Epidémiologie et Pathologie parasitaires», Faculté de Médecine de Paris-St-Antoine, 27, rue de Chaligny, F 75012 Paris

\section{Résumé}

Dans ce travail, nous étudions le comportement de Trichines adultes, extraites de l'intestin de souris infestées depuis 10 et 18 jours, puis transplantées dans l'intestin grêle de souris saines, par lots de 10 vers.

$1^{\circ}$ Dans le premier lot (souris infestées par des Trichines de 10 jours), nous avons constaté :

a) la présence des Trichines dans l'intestin jusqu'au $33^{\circ}$ jour suivant l'intervention.

Dans les muscles ont été récupérées des larves en quantité variable. Dans tous les sérums, les réponses immunologiques ont été négatives en immunofluorescence.

$2^{\circ}$ Dans le deuxième lot (souris infestées par des Trichines adultes âgées de 18 jours), nous n'avons observé ni vers intestinaux, ni larves musculaires chez aucune des 9 souris survivantes. De même, toutes les réactions immunologiques se sont révélées négatives.

Les faits essentiels constatés au cours de ces expérimentations consistent en l'allongement de la durée de survie des adultes par rapport aux infestations habituelles et dans l'incapacité des femelles âgées de 18 jours de pondre (contrairement à celles âgées de 10 jours). Ce stade le plus antigénique entraînant l'élimination des 
vers intestinaux se situe probablement lors de la maturation sexuelle ou au début de la ponte des larves.

Enfin, l'arrêt de la ponte à partir du $18^{\mathrm{e}}$ jour ne semble pas dû aux larves musculaires puisque nous avons constaté cet arrêt en dehors de toute présence concomitante de ces stades larvaires.

\section{Summary}

The purpose of this work was to study the behaviour of adult Trichinae taken out from the intestines of mice infected since 10 and 18 days, and transferred into the small intestine of healthy mice (10 worms).

\section{Mice infected with 10-day Trichinae.}

Trichinae were observed in the intestine up to the 33nd day after infection. Thus, total longevity of these worms has been of 43 days. Variable quantity of larvae was found in the muscles. Immune response was negative in all the sera (immunofluorescence).

\section{Mice infected with 18-day Trichinae.}

Neither intestinal worms, nor muscular larvae were found in any of the 9 surviving mice.

18-day females, though enclosing intra-uterine larvae seem unable to lay. That suggests that 18-day females have yet suffered the effect of the immune response of the host, without we could prejudge its mechanism: cellular or humoral.

So, essential facts observed during these experiments were :

1) The increase of the survival of the adults, compared with usual infections.

2) The incapacity of the 18-days females to lay (contrary to the 10-days). This most antigenic stage, which involves the elimination of intestinal worms probably occurs at the moment of sexual maturation or at the beginning of the larvae laid.

3) Muscular larvae do not seem responsible of the interruption of the laying, which happens without any concomitant presence of larval stages.

Dans nos publications antérieures nous avons étudié le devenir des larves musculaires de Trichinella spiralis introduites dans la cavité péritonéale et le déroulement de l'infestation intestinale chez des animaux normaux et des animaux soumis à des traitements immunodépresseurs.

Nous avons ènsuite cherché à connaître le comportement de Trichines adultes 
d'âges différents, prélevées dans l'intestin de souris récemment infestées, et transplantées dans l'intestin grêle de souris saines.

\section{Protocole expérimental.}

I. - Des souris sont infestées par ingestion de viande trichinée. Au dixième et au dix-huitième jour de l'infestation les souris sont tuées, les intestins sont prélevés et placés dans de l'eau physiologique à $37^{\circ} \mathrm{C}$. Ils sont alors ouverts et la muqueuse est raclée à l'aide d'une lame de verre. Les Trichines adultes sont recueillies par la méthode d'extraction de Baermann, elles sont lavées dans de l'eau physiologique à $37^{\circ} \mathrm{C}$ puis réparties par lots d'une dizaine de vers dans un volume de $0,2 \mathrm{ml}$ d'eau physiologique.

II. - Les souris saines réceptrices sont anesthésiées au chloral à 8 pour 1000 . Une incision abdominale médiane permet d'injecter, sous le contrôle de la vue, un lot de 10 Trichines adultes dans l'intestin grêle. La prévention d'accidents septiques est assurée par l'introduction d'antibiotiques dans la cavité péritonéale et le lavage de la suture pariétale par la même solution d'antibiotiques. Nous préparons ainsi deux lots de 20 souris ;

- le premier reçoit des Trichines adultes âgées de 10 jours,

- le second des Trichines adultes âgées de 18 jours.

III. - De 16 à 33 jours après cette inoculation intra-intestinale les souris réceptives sont sacrifiées et les larves musculaires de Trichine sont extraites par digestion artificielle dans le milieu: pepsine $(0,5$ pour 100$)$ - acide chlorhydrique (1 pour 100) à $37^{\circ} \mathrm{C}$.

IV. - La recherche des anticorps anti-trichines est faite par la réaction d'immunofluorescence sur des coupes à congélation de larves de Trichines musculaires. Le sérum d'une souris non infestée est pris comme témoin négatif et celui d'une souris trichinée positif au $1 / 160^{\circ}$ est utilisé comme témoin positif.

\section{Résultats.}

1. - Dans les deux lots de souris opérées, la mortalité est importante dans les premiers jours. Il s'agit très probablement d'infection ou de choc post-opératoire.

2. - Dans le premier lot, celui des souris ayant reçu des Trichines adultes de 10 jours, l'autopsie permet de récolter les larves intra-musculaires et les trichines adultes tandis que l'immuno-fluorescence est pratiquée sur le sérum.

Dans cette série toutes les souris examinées hébergent des larves musculaires retrouvées après digestion artificielle. Il faut en conclure que les Trichines adultes transplantées étaient en état de poursuivre leur ponte. Dans l'intestin nous avons retrouvé des femelles adultes aux $18^{\circ}, 29^{\circ}$ et $33^{\circ}$ jour après l'inoculation. Elles étaient donc âgées res- 


\begin{tabular}{|c|c|c|c|c|c|c|c|c|c|c|}
\hline $\begin{array}{l}\text { Nombre } \mathrm{de} \text { jours } \\
\text { après la transplanta- } \\
\text { tion } \ldots \ldots \ldots \ldots \ldots \ldots\end{array}$ & 16 & 17 & 18 & 29 & -7 & 29 & 29 & 29 & 33 & 33 \\
\hline $\begin{array}{l}\text { Taux d'anticorps fluo- } \\
\text { rescents } \ldots \ldots \ldots \ldots \ldots\end{array}$ & - & - & - & 0 & 0 & 0 & 0 & 0 & 0 & 0 \\
\hline $\begin{array}{l}\text { Nombre de larves } \\
\text { dans le diaphragme. }\end{array}$ & 8 & 7 & 2 & 7 & 60 & 4 & 2 & 1 & 50 & 3 \\
\hline $\begin{array}{l}\text { Nombre de larves } \\
\text { dans la totalité de la } \\
\text { masse musculaire .. }\end{array}$ & 40 & 29 & 12 & 30 & 400 & 18 & 10 & 2 & 300 & 25 \\
\hline $\begin{array}{l}\text { Nombre d'adultes } \\
\text { dans l'intestin (Baer- } \\
\text { mann) } \ldots \ldots \ldots \ldots \ldots\end{array}$ & 0 & 0 & 1 & 1 & 0 & 1 & 0 & 0 & 1 & 0 \\
\hline
\end{tabular}

pectivement de 28 jours, 39 jours et 43 jours. Nous n'avons pas trouvé de mâles mais ceux-ci étaient en très faible proportion au départ.

Dans un travail antérieur portant sur un grand nombre de souris infestées normalement par voie buccale, nous constations la disparition des vers adultes après le $35^{\circ}$ jour. Il semble donc qu'il y ait une survie prolongée des Trichines adultes dans la présente série d'expériences. Pour expliquer ce phénomène on peut penser que dans cette expérience, le stade antigénique est supprimé et qu'il n'y a pas de réponse immunitaire. Les phénomènes immunologiques responsables du rejet des adultes seraient déclenchés par un stade antigénique se situant avant la ponte des larves. Ce stade pourrait être celui de la maturation sexuelle.

Ce ne sont pas les larves des stades II et III (Berntzen, 1965) qui sont le stade antigénique puisqu'elles envahissent très vite les muscles et s'y développent. La réponse en immuno-fluorescence recherchée sur les sérums de souris chez lesquelles nous avons transplanté des T.S. vers adultes âgés de 10 jours et hébergeant de nombreuses larves musculaires est restée constamment négative, dans tous les cas, alors qu'une souris témoin, infestée par voie buccale avait un taux d'anticorps de $1 / 160^{\circ}$.

3. - Dans le second lot, celui des souris ayant reçu des Trichines adultes âgées de 18 jours, nous avons sacrifié les 9 animaux survivants au $29^{\circ}$ jour.

Les réactions d'immunofluorescence ont toutes été négatives.

Nous n'avons retrouvé ni un adulte dans l'intestin, ni une larve dans la totalité de la masse musculaire. Ceci contraste avec la première série dans laquelle, au contraire, tous les animaux étaient positifs.

Les Trichines adultes, âgées de 18 jours, quoique contenant encore de nombreuses larves dans leur utérus, paraissent donc incapable de les pondre. L'émission des larves du stade I par les femelles semble donc limitée dans le temps. Les femelles âgées de 18 jours paraissent avoir déjà subi les effets de la réponse immunitaire du $1^{\text {er }}$ hôte, sans qu'il soit possible de préjuger le mécanisme cellulaire ou humoral de celle-ci.

Nous avons constaté que les Trichines femelles prélevées au $18^{\circ}$ jour sont parfaite- 
ment vivantes, que l'utérus contient des œufs et des larves bien mobiles, mais que la vulve paraît obstruée par un précipité.

Nous rejoignons donc les conclusions de Denham (1966) qui pense que le stade adulte chez $T$. spiralis est indispensable au développement de l'immunité. Les résultats de notre expérimentation semblent s'opposer, par contre, à ceux obtenu par Despommier (1971) qui pense que ce sont les stades larvaires II et III qui sont responsables de l'immunité de réinfestation.

\section{Conclusions.}

Les transplantations de Trichines adultes chez une souris saine montrent des modifications dans le comportement de ce parasite. Ces modifications consistent en un allongement de la durée de survie du ver adulte dû à l'absence de réponse immunitaire (ce qui donne à penser que le stade antigénique est éliminé dans cette expérience) et une incapacité de pondre des femelles de Trichinella spiralis transplantées au $18^{\circ}$ jour (probablement parce qu'elles ont déjà subi une agression immunitaire) alors que celles qui sont transplantées au $10^{\circ}$ jour continuent de pouvoir le faire normalement.

\section{Bibliographie}

BERNTZEN (A. K.), 1965. - Comparative growth and development of Trichinella spiralis in vitro and in vivo, with a redescription of the life cycle. Exp. Parasit., XVI, 74-104.

Campbell (C. H.), 1955. - The antigenic role of the excretions and secretions of adult Trichinella spiralis in the production of immunity in mice. J. Parasit., XLIII, 593598.

Denham (D. A.), 1966. - Immunity to Trichinella spiralis. I. The immunity produced by mice in the four days of the intestinal phase of the infection. Parasit., LVI, 323-327.

-, 1966. - Immunity to Trichinella spiralis. II. Immunity produced by the adult worm in mice. Ibid., LVI, 745-751.

Despommier (D. D.), 1971. - Immunogenicity of the new born larva of Trichinella spiralis. J. Parasit., LVII (3), 531-534.

JACKSON (G. J.), 1959. - Fluorescent antibody studies of Trichinella spiralis. J. Infect. Dis., CV, 97-117.

Lancastre (F.), Mougeot (G.), Bazin (J. C.) et Depernet (D.), 1971. - Trichinose expérimentale. Deuxième note. Longévité et distribution des Trichines adultes chez les souris normales et immunotolérantes. Ann. Parasitol. Hum. comp., XLVI, 709-718.

Mulls (C. K.) et KeNT (N. H.), 1965. - Excretions and secretions of Trichinella spiralis and their role on immunity. Exp. Parasit., XVI, 300-310.

NoLF (L. O.), 1937. - The transplantation of gravid Trichinella spiralis. J. Parasit., XXIII, 574.

PAWLOWSKI (Z.) et RAUHUT (W.), 1971. - Transplantation of Trichinella spiralis in rats. Wiadom. Parazyt., XVII (56), 497-501. 